\section{Das Rekruitment immun- kompetenter Zellen in eine transplantierte Lunge ist trotz inkompletter Reinnervierung nicht behindert}

A. Lührmann ${ }^{1}$, R. V. Haberberger ${ }^{2}$, C. Mörike ${ }^{3}$, G. Steinhoff ${ }^{3}$, N. Krug ${ }^{4}$, R. Pabst ${ }^{1}$, T. Tschernig ${ }^{1}$

${ }^{1}$ Abteilung für Funktionelle und Angewandte Anatomie, Medizinische Hochschule Hannover

2 Institut für Anatomie und Zellbiologie, Justus-Liebig-Universität, Gießen

${ }^{3}$ Abteilung für Thorax-, Herz- und Gefäßchirurgie, Medizinische Hochschule Hannover

${ }^{4}$ Abteilung für Pneumologie, Medizinische Hochschule Hannover
Zusammenfassung: Hintergrund: Es ist unklar, ob der chirurgische Eingriff der Transplantation mit Durchtrennung sowohl der vegetativen Innervation als auch der Lymphgefäße und Bronchialarterien zu einer Veränderung von Immunreaktionen führt. Im Tierversuch sollte geklärt werden, ob eine induzierte Immmunreaktion nach einer Lungentransplantation uneingeschränkt ablaufen kann. Das Rekruitment von Immunzellen und der Innervierungszustand der Lungen sollte beurteilt werden. Methode: Die Untersuchungen erfolgten im syngenen Rattenmodell vier Monate nach einer orthotopen, linksseitigen Lungentransplantation ohne Abstoßungsreaktion und ohne immunsuppressive Behandlung. Bei einem Teil der Tiere wurden Leukozyten des Spenders und Empfängers differenziert. Nach Induktion einer pulmonalen Immunreaktion durch Ovalbumin (OVA) wurden die Zellen der Bronchoalveolären Lavage (BAL) und des Lungeninterstitiums für die Quantifizierung und Typisierung von Zellpopulationen immunzytologisch markiert und mittels Durchflusszytometer bzw. im Lichtmikroskop ausgewertet. Dabei wurde die rechte Kontrolllunge mit der linken transplantierten Lunge verglichen. Die Beurteilung des Innervierungszustandes erfolgte durch indirekte Immunfluoreszenz auf Gefrierschnitten. Ergebnisse: Die Gesamtzellzahl der Leukozyten in der transplantierten Lunge ist nicht vermindert. Die Population der $\mathrm{CD}^{+}$T-Zellen in der BAL der Transplantatlunge ist gegenüber der Kontrollseite signifikant erhöht. Die Lunge ist partiell reinnerviert und beinhaltet in der BAL vereinzelt ortsständige, spenderspezifische Leukozyten. Schlussfolgerungen: Das Rekruitment von Abwehrzellen in die transplantierte Lunge nach induzierter Immunreaktion ist nicht eingeschränkt. Eine unvollständige Reinnervierung hat keinen Einfluss auf eine zelluläre Entzündungsreaktion.

The Recruitment of Immune Cells into a Grafted Lung is not Impaired in Spite of Incomplete Reinnervation: Background: It is not clear whether surgical intervention during lung transplantation which includes cutting vegetative nerves, lymphatic vessels and bronchial arteries, leads to alterations in immune responses. Thus, it was studied in an animal model whether an induced pulmonary immune reaction after syngenic lung transplantation was impaired without the influence of immunosuppression and rejection. The recruitment of leukocytes and the status of reinnervation was examined. Methods: Syngenic transplantation of the left lung was performed in Le-

Pneumologie 2000; 54: 564-568

(c) Georg Thieme Verlag Stuttgart · New York ISSN 0934-8387 wis rats without rejection and therefore without immunosuppressive therapy. In a subgroup of animals host and donor leukocytes were distinguished. An ovalbumin (OVA)-specific pulmonary immune response was induced four months after transplantation. Bronchoalveolar lavage (BAL) and interstitial leukocytes were examined using flow cytometry and immunocytology, comparing the right lung and the grafted left lung. Immunohistology was performed to detect nerve fibers on cryostat sections. Results: An induced cellular inflammation was observed in the right host lung as well as in the grafted left lung. However, the CD4 T cell numbers in the BAL were increased in the left lung. Single donor-type leukocytes could still be observed four months after transplantation. A partial reinnervation was found. Conclusions: The recruitment of immune cells into the lung interstitium and bronchoalveolar space of grafted lungs is not impaired. The incomplete reinnervation has no influence on leukocyte recruitment.

\section{Einleitung}

Der chirurgische Eingriff der Transplantation ist verbunden mit der Durchtrennung der Lymphgefäße, der Bronchialarterien und der Unterbrechung der vegetativen Innervation. Nur wenig ist bekannt, inwieweit die entstandene Gewebeschädigung nach Ischämie und Reperfusion oder die komplette Denervierung Einfluss auf den Ablauf einer Immunreaktion in der transplantierten Lunge haben. Das Immunsystem steht in enger Verbindung zum Nervensystem. In den Luftwegen sind an der Interaktion die Nervenfasern und die Zellen des Immunsystems, z. B. Makrophagen, Mastzellen und Granulozyten, beteiligt [1]. Eine transplantationsbedingte Denervierung könnte die zelluläre Immunreaktion in der Lunge beeinträchtigen. Im vorliegenden Versuch wurde vier Monate nach einer syngenen Lungentransplantation eine pulmonale Immunreaktion durch OVA induziert. Mit einer seitengetrennten Analyse konnte zwischen der transplantierten, linken Lunge und der rechten Lunge unterschieden werden. Zur Differenzierung zwischen Empfänger- und Transplantatzellen wurden zum Teil Rattenstämme verwendet, die sich im CD45-Molekül (RT7-System) unterscheiden, ohne eine Abstoßung herbeizuführen [2,3]. Zur Erkennung des Innervationszustandes wurde durch indirekte Immunfluoreszenz das Neuropeptid Calcitonin Gene Related Peptide (CGRP) nachgewiesen.

Die Arbeit stellte folgende Fragen: Ist das Rekruitment von Abwehrzellen in die transplantierte Lunge nach induzierter Immunreaktion eingeschränkt? In welchem Umfang sind die 
Leukozyten des Empfängers in die transplantierte Lunge eingewandert? Wie ist der Innervationszustand vier Monate nach Transplantation?

\section{Material und Methoden}

Für die Versuche wurden sechs 10-12 Wochen alte männliche Lewis-Ratten vom Stamm RT7a und RT7b verwendet. Lewis-Ratten wurden linke Lungen orthotop transplantiert. Ausführlich wurde dies von Prop et al. [4] beschrieben. Hier in Kürze: Nach linksseitiger Thorakotomie wurde die linke Lunge des Spendertieres (RT7a: $n=3$; RT7b: $n=3$ ) entnommen, die Ischämiezeit lag bei etwa einer halben Stunde. Nach Entfernung der Lunge im Empfängertier (RT7a: $n=6$ ), wurde durch Anastomosen der Arteria und Vena pulmonalis und des Bronchus die Spenderlunge eingesetzt.

Nach vier Monaten wurden den Versuchstieren zur Sensibilisierung (genaue Beschreibung durch Schuster et al. [5]) je $1 \mathrm{mg}$ OVA subkutan injiziert. Gleichzeitig erfolgte eine intraperitoneale Verabreichung von $5 \times 10^{9}$ hitzegetöteten Bordetella pertussis Bakterien. Nach 7-8 Tagen wurde der Vorgang wiederholt. Nach weiteren 6-7 Tagen inhalierten die Tiere für 15 min eine $5 \%$ ige OVA/Kochsalz-Lösung. $22 \pm 2$ Stunden nach der Provokation erfolgte die Tötung der Tiere durch Aortenpunktion in Narkose. Für die sich anschließende seitengetrennte BAL wurde nach Kanülierung der Trachea mittels Knopfkanüle und Präparation des Lungenhilus zunächst der rechte Hauptbronchus abgeklemmt und linksseitig mit $10 \times 2 \mathrm{ml} \mathrm{NaCl} \mathrm{0,9 \%} \mathrm{gespült.} \mathrm{Danach} \mathrm{erfolgte} \mathrm{die} \mathrm{Spülung} \mathrm{des}$ rechten Lungenflügels mit $10 \times 3 \mathrm{ml}$. Die seitengleichen Fraktionen wurden vereinigt. Für die Kryokonservierung wurden Teile der Lunge mit OCT gefüllt und in flüssigem Stickstoff eingefroren [5]. Durch mechanische Behandlung konnte die restliche Lunge zu einer Einzelzellsuspension aufgearbeitet werden. Aus den Zellsuspensionen von BAL und Lungenhomogenat wurde mittels einer Zytozentrifuge Zytospinpräparate hergestellt. Zelldifferenzierung erfolgte nach Färbung mit May-Grünwald/Giemsa-Reagenz. RT7b-positive und -negative Zellen wurden auf Zytospotpräparaten der BAL nach Antikörperfärbung im Lichtmikroskop unterschieden [6]. Die Detektion von $\mathrm{CD}^{+}$, $\mathrm{CD}^{+}$T-Zellen und NK-Zellen erfolgte im Durchflusszytometer (ausführlich bei Fliegert et al. [7]). Da rechte und linke Lunge unterschiedlich groß sind, wurden die Zellen aus der BAL und des Lungeninterstitiums von rechter Kontrollseite und dem Spenderorgan in relativen Anteilen angegeben. Wenn die Zellzahlen der linken Lunge auf das Volumen der rechten Lunge hochgerechnet werden, ergeben sich die gleichen Ergebnisse wie für den Vergleich der prozentualen Werte.

Für den Neuropeptidnachweis (ausführlich bei Haberberger et al. [8]) wurde an einem Kryostaten von den Lungen Sagittalschnitte einer Dicke von $12 \mu \mathrm{m}$ angefertigt, fixiert und mit CRGP-spezifischem Antiserum immunhistologisch gefärbt. Die Schnitte wurden mit einem Fluoreszenzmikroskop (Anregungsfilter 525-560 nm, Sperrfilter 570-650 nm) analysiert. Die semiquantitative Auswertung erfolgte an drei Schnitten (Mindestabstand $120 \mu \mathrm{m}$ ) pro transplantierter und nicht-transplantierter Lunge durch zwei Untersucher. Für die Kontrolle wurde die Innervation an Gewebe von Tieren 14 Tage nach Transplantation bestimmt, die aus vorangegangenen Versuchen zur Verfügung standen.
Zur statistischen Auswertung wurde das Programm SPSS für Windows 6.0.1. verwendet. Ermittelt wurden Mittelwerte und Standardfehler der Versuchsgruppen. Zum Nachweis von signifikanten Unterschieden wurden die einzelnen Gruppen gegeneinander mit Hilfe des Wilcoxon-Testes für zwei verbundene Stichproben verglichen. Ein $\mathrm{p} \leq 0.05$ wurde als statistisch signifikant angesehen.

\section{Ergebnisse}

Nach Transplantation, Sensibilisierung und pulmonaler Antigenprovokation konnten bei den Versuchstieren keine sichtbare Krankheitszeichen festgestellt werden. Das Gewebe der rechten und linken, transplantierten Lunge zeigte sich bei der Präparation makroskopisch unauffällig.

Der Einstrom der Leukozyten in die transplantierte Lunge war nicht vermindert. Bei den CD4+ $4^{+}$-Zellen gab es einen signifikanten Anstieg in der BAL des Transplantats. Die Anteile der Alveolarmakrophagen, der neutrophilen Granulozyten, der Lymphozyten insgesamt und der $\mathrm{CD}^{+} \mathrm{T}$-Zellen waren $z w i-$ schen rechter und linker Lunge nicht signifikant verändert (Abb.1). Der Vergleich der Zellen im Lungeninterstitium von beiden Lungen ergab keine Abweichungen bei der Untersuchung der NK-Zellen, der Lymphozytenpopulation insgesamt, der $\mathrm{CD}^{+}{ }^{+}$und $\mathrm{CD} 8^{+}$T-Zellen (Abb. 2).

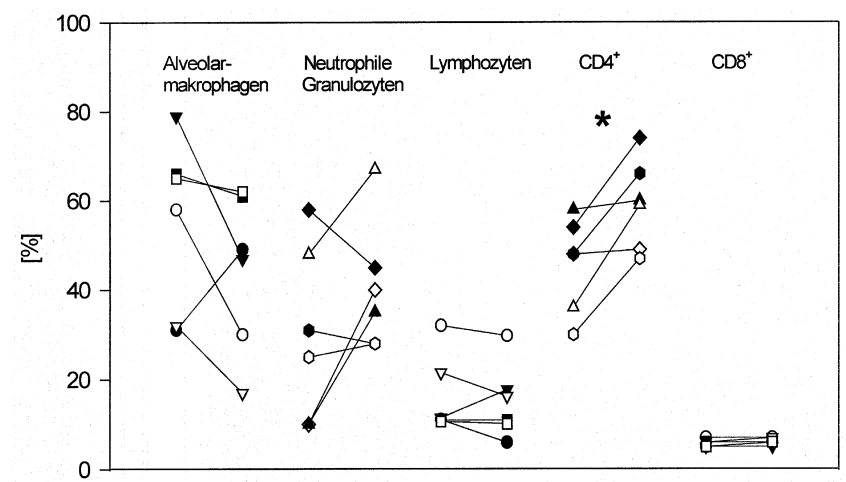

Abb. 1 Die transplantierte Lunge zeigte eine normale Entzündungsreaktion. Beim Vergleich von Kontrolllunge (links) und Spenderlunge (rechts) zeigte sich in der BAL ein signifikanter Unterschied der CD4 ${ }^{+}$ T-Zell-Population. Die Symbole kennzeichnen verschiedene Tiere. Signifikante Unterschiede sind mit ${ }^{*}$ gekennzeichnet $\left({ }^{*} \mathrm{p} \leq 0,05\right.$; Wilcoxon-Test).

Durch immunzytologische Färbung der BAL konnten Leukozyten vom Empfänger- und Spendertyp differenziert werden. Einzelne ortsständige Zellen vom Spendertyp waren noch nach vier Monaten nachweisbar (Abb. 3).

Für die Darstellung der nervalen Innervationssituation wurde das Neuropeptid CGRP nachgewiesen. Postoperativ sind nach 14 Tagen keine CGRP-haltigen Nervenfasern im Gewebe zu sehen (Abb.4a). Nach vier Monaten ist eine partielle Reinnervierung zu erkennen (Abb. $\mathbf{4 b}$ ). 


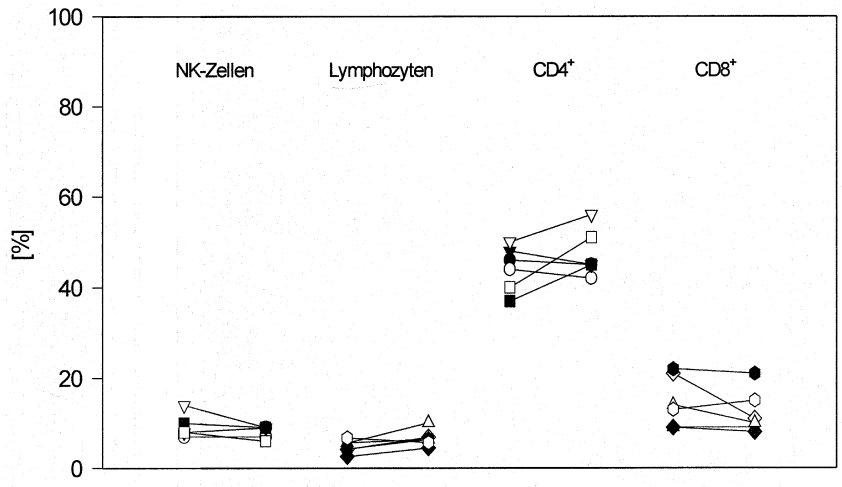

Abb. 2 Der Vergleich Kontrolllunge (links) und transplantierte Lunge (rechts) im Interstitium ergab in keiner untersuchten Zellpopulation einen signifikanten Unterschied. Die Symbole kennzeichnen verschiedene Tiere.

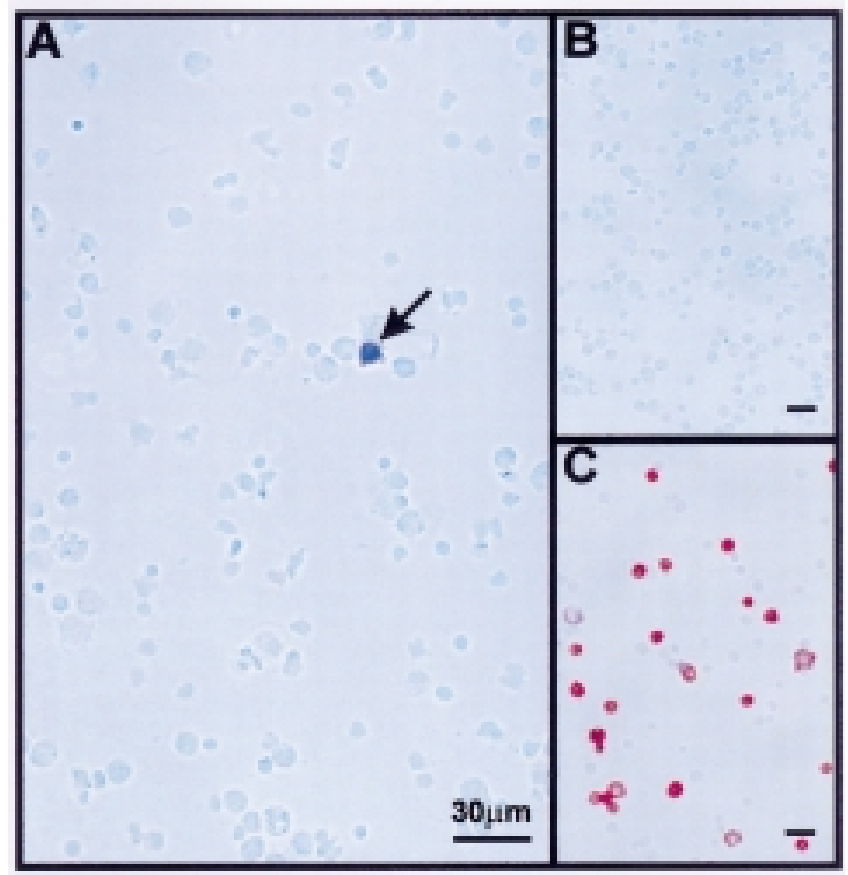

Abb. 3 Mikroskopische Aufnahmen von Zytospinpräparaten mit Zellen der BAL nach Färbung mit einem spenderspezifischen Antikörper. Vier Monate nach Transplantation (Abb. A) besitzen nur noch vereinzelte Zellen die Spezifität des Spendertieres (blau), während diese Zellen 14 Tage nach Transplantation deutlich häufiger vorkommen (rot) (Abb.C). Die Kontrolllunge zeigt keine positiven Zellen (Abb. B).

\section{Diskussion}

Durch eine aktive Sensibilisierung kommt es nach der Provokation mit OVA zunächst zu einer Ag-Ak-Komplexbildung in der Lunge. Das Komplementsystem wird aktiviert und führt zur chemotaktischen Anlockung von Immunzellen wie z.B. Granulozyten und Makrophagen [9]. Dieses bestätigte sich durch die vorliegende Untersuchung. Der Anstieg der Lymphozytenzahl entspricht anderen Ergebnissen nach OVAProvokationen [5].
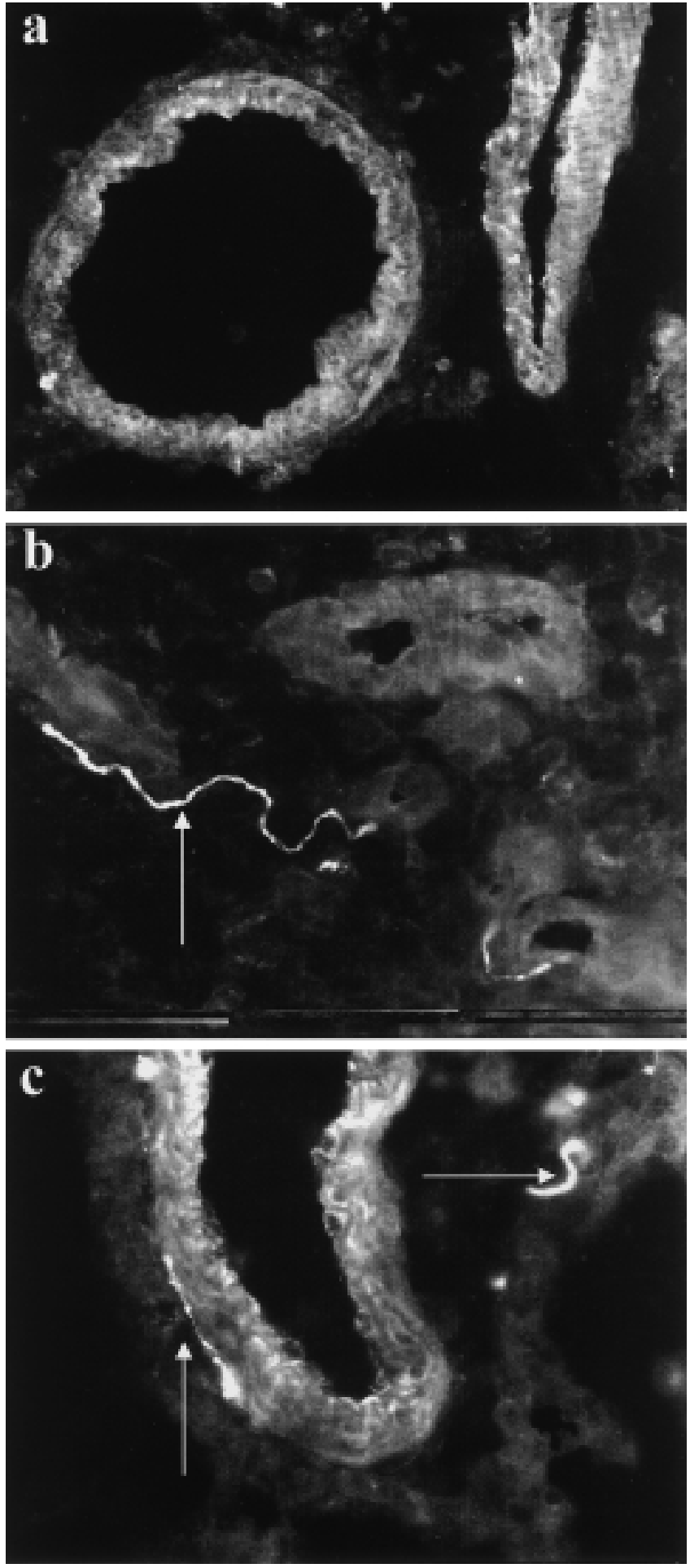

Abb. 4 Fluoreszenzmikroskopische Aufnahme (400fache Vergrößerung) von Gewebeschnitten nach Kryokonservierung und immunhistochemischer Färbung mit einem CGRP-spezifischen Antikörper. (a) Lungengewebe 14 Tage nach Transplantation zeigt keine positive Färbung; die Lunge ist denerviert. (b) Lungengewebe vier Monate nach Transplantation zeigt eine Reinnervierung (Pfeil). (c) Innervierungszustand (Pfeil) der rechten Kontrolllunge vier Monate nach Transplantation. 
Die Transplantation hat die Reaktion auf OVA nur in einem der untersuchten Parameter beeinflusst. Der Vergleich von Lymphozytenzahlen der rechten und der transplantierten Lunge zeigte einen Anstieg der $\mathrm{CD}^{+}$T-Zellen in der BAL. Curtis et al. [10] zeigten im Mausmodell, dass es nach einer Sensibilisierung und Provokation mit Schaferythrozyten zu einem starken Influx von $\mathrm{CD}^{+}{ }^{+}$T-Zellen kommt. Welchen Einfluss die Transplantation auch noch vier Monate später auf eine Immunantwort hat, kann nicht abschließend geklärt werden. An der Migration von Lymphozyten und deren Regulation ist eine Vielzahl von Chemokinen beteiligt [11].

Dass die Leukozyten in der BAL 4 Monate nach Transplantation zum größten Teil die Spezifität des Empfängertieres besitzen, ergänzt frühere Untersuchungen. Schon 14 Tage nach Transplantation waren noch $30 \%$ ursprüngliche Lymphozyten [12] zu finden, nach weiteren 14 Tagen waren bei jedem Tier nur noch einzelne Spenderzellen nachweisbar [6].

Inwieweit die Reinnervierung bei der Regulation einer Immunantwort eine Rolle spielt ist noch ungeklärt. Im Bereich der sensorischen, afferenten Nerven sind die marklosen CFasern zu finden. Sie liegen innerhalb des Lungenepitheliums und enthalten mit Neuropeptiden gefüllte Vesikel. Die Neuropeptide beinhalten verschiedene Tachykine, wie z.B. Substanz P, CGRP und Neurokinin A [1]. Die tachykinhaltigen CFasern konnten im Bereich der humanen Bronchien, der Bronchiolen und den distalen Bereichen der Atemwege identifiziert werden. Inwieweit die Tachykine in den Alveolen vorhanden sind, konnte dagegen noch nicht abschließend geklärt werden $[13,14]$. Histologische Studien zeigten, dass Nervenfasern durch Tachykine z.B. Substanz P und CGRP vermittelt, mit verschiedenen Zellen des Immunsystems in Kontakt treten [15]. Mastzellen gelten als „Informationswandler" zwischen nervalen Reizen und immunologischen Ereignissen [16]. Zusammen mit den Makrophagen kleiden sie die Muskelschicht des Respirationstraktes aus und sind in räumlicher Nähe zu Substanz P- und CGRP-haltigen Nervenfasern anzutreffen [17]. Neuropeptide und neurotrophe Faktoren (z.B. NGF) sind in der Lage, die Freisetzung von Mediatoren in den Mastzellen zu induzieren [18,19].

Durch die Transplantation war eine vollständige Denervation der Lunge gewährleistet. Erst nach acht Monaten kommt es bei der Ratte laut Kawaguchi et al. [20] zu einer Wiederherstellung der nervalen Versorgung. Buvry et al. [21] konnten eine partielle Reinnervation der Rattenlunge nach 5 Monaten durch den Nachweis von neugebildeten CGRP-haltigen Nervenfasern zeigen. Informationen über eine mögliche Reinnervierung und deren zeitlichen Ablauf in menschlichen Lungentransplantaten gibt es bisher nicht. Im vorliegenden Versuch konnte durch den immunhistologischen Nachweis des Neuropeptides CGRP gezeigt werden, dass zumindest eine partielle Reinnervierung erfolgt ist. Es muss dabei beachtet werden, dass sich diese qualitativ und quantitativ von der ursprünglichen Innervation unterscheidet. Die unvollständige Reinnervierung und damit verbundene Unterbrechung der Wechselwirkungen von Immun- und Nervensystem, könnte die Ursache dafür sein, dass es zu unterschiedlich starken Reaktionen auf ein Antigen kommt. Ein direkter Zusammenhang zwischen dem Immun- und Nervensystem kann allerdings nicht hergestellt werden.
Das Rekruitment von Abwehrzellen in die transplantierte Lunge nach induzierter Immunreaktion ist nicht eingeschränkt. Eine unvollständige Reinnervierung hat keinen Einfluss auf eine Entzündungsreaktion.

\section{Danksagung}

Dieses Projekt wurde durch die DFG (Pa 240/8-1) gefördert. Die Autoren möchten Herrn Dr. Wonigeit, Medizinische Hochschule Hannover für die Tiere, K. Westermann und A. Weiß für die technische Mitarbeit, D. Stelte für Erstellung der Fotos und S. Fryk für die Korrektur des Manuskriptes danken.

\section{Literatur}

${ }^{1}$ James DE, Nijkamp FP. Neuro-immune interactions in the lung. Clin Exp Allergy 1999; 29: 1309-1319

${ }^{2}$ Wonigeit K. Characterization of the RT-Ly-1 and RT-Ly-2 alloantigenic systems by congenic rat strains. Transplant Proc 1979; 11: $1631-1635$

${ }^{3}$ Kampinga J, Kroese FG, Pol GH et al. RT7-defined alloantigens in rats are part of the leucocyte common antigen family. Scand J Immunol 1990; 31: 699-710

${ }^{4}$ Prop J, Ehrie MG, Crapo JD, Nieuwenhuis P, Wildevuur CR. Reimplantation response in isografted rat lungs. Analysis of causal factors. J Thorac Cardiovasc Surg 1984; 87: 702 - 711

${ }^{5}$ Schuster M, Tschernig T, Krug N, Pabst R. Lymphocytes migrate from the blood into the bronchoalveolar lavage and lung parenchyma in the asthma model of the Brown Norway rat. Am J Respir Crit Care Med 2000; 161: 558-566

${ }^{6}$ Tschernig T, Heizmann O, Boeke K, Steinhoff G, Wonigeit K, Pabst $\mathrm{R}$. Macrophages and lymphocytes in the bronchoalveolar space have different turnover times as shown by experimental lung transplantation. Am J Respir Crit Care Med 1998; 157: A812

${ }^{7}$ Fliegert FG, Tschernig T, Pabst R. Comparison of lymphocyte subsets, monocytes, and NK cells in three different lung compartments and peripheral blood in the rat. Exp Lung Res 1996; 22: 677-690

${ }^{8}$ Haberberger R, Schemann M, Sann H, Kummer W. Innervation pattern of guinea pig pulmonary vasculature depends on vascular diameter. J Appl Physiol 1997; 82: 426-434

${ }^{9}$ Baumann U, Kohl J, Tschernig T et al. A codominant role of Fc gamma RI/III and C5aR in the reverse Arthus reaction. J Immunol 2000; 164: 1065-1070

${ }^{10}$ Curtis JL, Byrd PK, Warnock ML, Kaltreider HB. Requirement of CD4-positive T cells for cellular recruitment to the lungs of mice in response to a particulate intratracheal antigen. J Clin Invest 1991; 88: $1244-1254$

${ }^{11}$ Wells TN, Proudfoot AE. Chemokine receptors and their antagonists in allergic lung disease. Inflamm Res 1999; 48: 353-362

12 Tschernig T, Boeke K, Steinhoff G, Wonigeit K, Pabst R, Westermann J. The lung as a source and a target organ for T- and Blymphocytes. Am J Respir Cell Mol Biol 1997; 17: 414 - 421

${ }^{13}$ Lundberg JM, Hokfelt T, Martling CR, Saria A, Cuello C. Substance P-immunoreactive sensory nerves in the lower respiratory tract of various mammals including man. Cell Tissue Res 1984; 235 : $251-261$

${ }^{14}$ Komatsu T, Yamamoto M, Shimokata K, Nagura H. Distribution of substance P-immunoreactive and calcitonin gene-related peptide-immunoreactive nerves in normal human lungs. Int Arch Allergy Appl Immunol 1991; 95: 23-28

${ }^{15}$ Dimitriadou V, Lambracht-Hall M, Reichler J, Theoharides TC. Histochemical and ultrastructural characteristics of rat brain perivascular mast cells stimulated with compound 48/80 and carbachol. Neuroscience 1990; 39: 209-224 
${ }^{16}$ Williams RM, Bienenstock J, Stead RH. Mast cells: the neuroimmune connection. Chem Immunol 1995; 61: $208-235$

${ }^{17}$ Church MK, Lowman MA, Robinson C, Holgate ST, Benyon RC. Interaction of neuropeptides with human mast cells. Int Arch Allergy Appl Immunol 1989; 88: 70-78

${ }^{18}$ Horigome K, Pryor JC, Bullock ED, Johnson EMJ. Mediator release from mast cells by nerve growth factor. Neurotrophin specificity and receptor mediation. J Biol Chem 1993; 268: 14881-14887

19 Piotrowski W, Devoy MA, Jordan CC, Foreman JC. The substance $P$ receptor on rat mast cells and in human skin. Agents Actions 1984; $14: 420-424$

${ }^{20}$ Kawaguchi AT, Shirai M, Yamano M, Ishibashi-Ueda H, Yamatodani A, Kawashima Y. Afferent reinnervation after lung transplantation in the rat. J Heart Lung Transplant 1998; 17: 341 - 348

21 Buvry A, Yang YR, Tavakoli R, Frossard N. Calcitonin gene-related peptide-immunoreactive nerves and neuroendocrine cells after lung transplantation in the rat. Am J Respir Cell Mol Biol 1999; 20: $1268-1273$

\section{A. Lührmann}

Abteilung Funktionelle und Angewandte Anatomie 4120 Medizinische Hochschule Hannover

30623 Hannover

E-mail: Luehrmann.Anke@mh-hannover.de
„Forschungsstipendium Lungenfibrose“ der Deutschen Lungenstiftung \& Stiftung zur Erforschung der Lungenfibrose Waldhof Elgerhausen

Diagnostik, Klassifizierung und Therapie der Lungenfibrose sind aus wissenschaftlicher und klinischer Sicht unbefriedigend. Selbst die histologische Diagnose ist selten hilfreich für eine weiter erfolgreiche Therapie. Wir planen eine mehrjährige Analyse von Krankheitsverläufen an einem großen Krankengut mehrerer Kliniken unter Berücksichtigung der histologischen Diagnose, der Behandlung mit Immunsuppressiva und der SauerstoffLangzeit-Therapie. Für das Projekt stehen zunächst 50000 DM zur Verfügung.

Gesucht wird eine jüngere klinische Forscherin/klinischer Forscher als zentrale Koordinatorin/zentraler Koordinator.

Einzelheiten zum Projekt und zur Bewerbung sind zu erfragen bei Prof. Dr. Helmut Fabel, Hannover, Tel.: 0511/ 9063347 oder Prof. Dr. Harald Morr, Greifenstein, Tel.: 06449/927261. 